\title{
Application of Ground Penetrating Radar Surveys and GPS Surveys for Monitoring the Condition of Levees and Dykes
}

\author{
Dariusz TANAJEWSKI and Mieczysław BAKUŁA \\ University of Warmia and Mazury in Olsztyn, Poland \\ e-mails: d.tanajewski@kgsin.pl (corresponding author); mbakula@uwm.edu.pl
}

\begin{abstract}
This paper analyses the possibility of using integrated GPS (Global Positioning System) surveys and ground penetrating radar surveys to precisely locate damages to levees, particularly due to the activity of small fossorial mammals. The technology of intercommunication between ground penetrating radar (GPR) and an RTK (Real-Time Kinematic) survey unit, and the method of data combination, are presented. The errors which may appear during the survey work are also characterized. The procedure for processing the data so that the final results have a spatial character and are ready to be implemented in digital maps and geographic information systems (GIS) is also described.
\end{abstract}

Key words: Ground Penetrating Radar surveys, GPS, RTK, levees monitoring, data synchronization.

\section{INTRODUCTION}

\subsection{Background}

Floods are frequent and devastating natural disasters. It is estimated that the effects of floods have affected almost 2.9 billion people in the last 30 years, of whom 550 thousand died, 360 thousand required hospitalization and 4.5 million lost their homes (Doocy et al. 2013). The calculation of the total

Ownership: Institute of Geophysics, Polish Academy of Sciences;

(C) 2016 Tanajewski and Bakuła. This is an open access article distributed under the Creative Commons Attribution-NonCommercial-NoDerivs license,

http://creativecommons.org/licenses/by-nc-nd/3.0/. 
economic and natural losses is practically impossible. One hundred major floods were recorded in Europe between 1998 and 2002 alone and insured economic losses totaling 25 billion euro (de Vries et al. 2003). Although this amount may be shocking, it was only a small fraction of the value of economic assets liable to flooding. The European Commission estimated in the Communication on "Flood risk management; flood prevention, protection and mitigation" (EC 2004) that land worth between 0.5 and 1 billion euro was located within $500 \mathrm{~m}$ from the European coastline. In the area liable to extreme flooding along the Rhine river, the potential losses could total another 165 billion euro.

The growing risk of extreme phenomena and potential financial losses forced the European Commission to take action aimed at flood prevention, protection and mitigation. Consultations with governments, nongovernmental organizations, scientists and people started to develop an interdisciplinary and comprehensive approach to flood risk management. As a result, "Directive on the assessment and management of flood risks" (EC 2007), which obliged member states to take preventive and protective action, including monitoring and maintenance of a good condition of levees and dykes, was established.

\subsection{Levee monitoring - technical aspects}

The most popular methods of examining the condition of levees are dynamic probing, geotechnical boreholes and laboratory analyses of the collected samples (Bruce 1993, Gołębiowski et al. 2012). However, they provide data only for a small ground volume. Borys (2013) and technical guidelines ("Guidelines for evaluations of the condition and safety of levees") recommend that ground samples ought to be collected in places where there is a suspicion of levee construction weakening, but not less frequently than from 3 to 5 samplings per $1 \mathrm{~km}$ of the checked structure, depending on the levee class and its subsurface type. This means that a correct levee stability evaluation depends to a large degree on the knowledge, experience and subjective assessment of the person who conducts the field reconnaissance. The human factor can be reduced by increasing the number of probings, but this rises the costs of work and weakens the levee crown.

An alternative to geotechnical levee examination methods is the ground penetrating radar method (commonly called GPR) (Lee et al. 2012, Gołębiowski et al. 2012, Di Prinzio et al. 2010, Song and Guo 2010, Mori 2009, Mydlikowski and Beziuk 2009, Mydlikowski and Szynkiewicz 2009, Szynkiewicz 2000). It allows a near-surface and non-invasive examination of the structure of hydraulic engineering facilities along their entire length and in a relatively short time. In some cases, the survey results are available 
in real time and can indicate the areas where the levee structure is weakened at the survey site. Such situations have been examined by the abovementioned researchers. However, frequently only office survey processing enables the location of dangerous loosenings. A problem with the indication of such places in the field then appears, particularly if several kilometers of a levee were the object of the survey. A comprehensive method for the acquisition of data on the technical condition of hydrotechnical structures, which will determine the location of anomalies in them in geographic space, is therefore needed. This task can be managed by an integrated survey set consisting of a GPS receiver and a ground penetrating radar. Both of these technologies have been designed for completely different purposes. However, in the context of monitoring the condition of levees, only their combination allows for receiving full information on the object of examination (Urbini et al. 2001).

The aim of the conducted research was to check the feasibility and evaluate the efficiency of integrated GPR and GPS surveys in detection and spatial location of damage to river embankments. The authors were particularly interested in the tunnels and burrows of small mammals which inhabit the levee crown, because their destructive activity weakens the levee and raises flood risk to the highest degree (Niederleithinger et al. 2008). Satellite measurements were also used to determine the levee crown height along the entire examined length. The results have practical application and can be used by public services dealing with water management and flood safety.

\section{INTEGRATED GPR AND GPS SURVEYS}

\subsection{GPR surveys: theoretical aspects}

Detection of objects by the GPR method is based on the principle of electromagnetic wave reflection on boundaries. The transmitting antenna emits single pulses into the examined medium where they reflect and disperse at levels with different lithology, porosity or density. The receiving antenna captures the reflected waves and records them in a digital format (Daniels 2000). The received radargrams reflect the internal composition of the examined structure (Karczewski et al. 2011, Jol 2009).

Subsurface components have dielectric properties and can often be described by relative electric permittivity $\varepsilon_{r}$ and electrical conductivity $\sigma$ (Jol 2009).

The values of these parameters play an important role in ground penetrating radar surveys, because attenuation $\alpha$ and electromagnetic wave velocity $v$ in a given medium are determined on their basis. Attenuation determines the antenna's depth range. The depth reached by the electromagnetic wave is not constant and depends on the degree of wave energy reduction. 
The factors attenuating signal energy are: high electrical conductivity of the examined medium, the presence of salts and clayey materials, and ground penetrating radar antenna frequency (Di Prinzio et al. 2010, Tillard and Dubois 1995, Smith and Jol 1995). Table 1 shows the values of relative permittivity and electromagnetic wave velocity for different media.

Table 1

Typical electromagnetic properties of the selected materials at $100 \mathrm{MHz}$ (Morey 1998)

\begin{tabular}{|l|c|c|c|c|}
\hline Material & $\begin{array}{c}\text { Relative dielectric } \\
\text { permittivity } \\
\varepsilon_{r}\end{array}$ & $\begin{array}{c}\text { Electrical } \\
\text { conductivity } \\
\sigma[\mathrm{mS} / \mathrm{m}]\end{array}$ & $\begin{array}{c}\text { Attenuation } \\
\alpha[\mathrm{dB} / \mathrm{m}]\end{array}$ & $\begin{array}{c}\text { Velocity } \\
v[\mathrm{~m} / \mathrm{ns}]\end{array}$ \\
\hline Air & 1 & 0 & 0 & 0.30 \\
Fresh water & 81 & 0.05 & 0.1 & 0.033 \\
Dry sand & $3-5$ & 0.01 & 0.01 & 0.15 \\
Saturated sand & $20-30$ & $0.1-1.0$ & $0.03-0.3$ & 0.06 \\
Silts & $5-30$ & $1-100$ & $1-100$ & 0.07 \\
Clays & $5-40$ & $2-1000$ & $1-300$ & 0.06 \\
\hline
\end{tabular}

However, the ground medium is usually not homogeneous and consists of several different materials. Relative dielectric permittivity is then not known and wave propagation velocity is measured at the site of the examination. Its value is estimated based on the measurement of an object which is at known depth. Another method is velocity profiling, called a wide angle reflection and refraction (WARR) or a common mid-point (CMP) (Daniels 2004, Fisher et al. 1992). It consists in increasing the distance of the receiving antenna from the transmitting antenna with simultaneous recording of the reflected signals. The relationship between the reflected wave transit time and the electromagnetic wave velocity in the medium can then be established (Karczewski et al. 2011):

$$
t_{r}=\frac{\sqrt{x^{2}+4 \cdot d}}{v},
$$

where $t_{r}$ is transit time to and from the target, $x$ distance between GPR antennas, $d$ depth to reflecting interface, and $v$ velocity of propagation.

Another velocity estimation method is fitting the arms of a synthetic hyperbola to the hyperbolas in the radargram. This velocity value is adopted for the one whose theoretical shape best correlates with the shape of the recorded hyperbolas. The correctly determined curve shape is created according to the formula (Sandmeier 2011): 


$$
t_{R}=\left[\sqrt{\frac{\left(x_{i}-x_{d}\right)^{2}}{v^{2}}+\left(\frac{t_{0}}{2}+\frac{r}{v}\right)^{2}}-\frac{r}{v}\right]+\left[\sqrt{\frac{\left(x_{i}+x_{d}\right)^{2}}{v^{2}}+\left(\frac{t_{0}}{2}+\frac{r}{v}\right)^{2}}-\frac{r}{v}\right],
$$

where $t_{R}$ is recording time of the signal reflected from the object in the position $x_{i}, t_{0}$ recording time of the signal reflected directly over the object, $x_{d}$ distance between the transmitting antenna and receiving antenna, $x_{i}$ antenna position relative to the object, $r$ object (conduit) radius, and $v$ wave velocity in the medium.

The formula shows that the diameter of the examined reference object and the distances between the transmitting and receiving antennas must be known in hyperbola shape determination. The fitting process itself is performed at the data processing stage. The depth determination error for this method is estimated at $10-12 \%$ of the real value (Jol 2009, Karczewski et al. 2011).

\subsection{GPS surveys: theoretical aspects}

The GPS survey method consists in the determination of the threedimensional position of the receiver in the global coordinate system, based on the propagation time of radio signals sent by satellites. Coordinate determination accuracy depends on the technical parameters of the receiver and the applied survey technology. Two-frequency receivers, operating in RTK (Real Time Kinematic) survey mode, are commonly used in surveying for precise GPS measurements. From the architectural point of view, this technology consists of: a base receiver set on a point with known coordinates, a rover receiver used to determine the coordinates of successive points, and a communication channel through which the base receiver sends its observations to the rover receiver. The accuracy of position determination by this method is $1-2 \mathrm{~cm}$ for the horizontal position and $5 \mathrm{~cm}$ for altitude over approximately $10 \mathrm{~km}$ of baseline separation (distance between the base station and the rover receiver) (Edwards et al. 2010). Rapid and very precise measurement depends on resolving the unknown cycle ambiguities of the doubledifferenced carrier phase data to integers (ambiguity is the unknown number of whole wavelengths of the carrier signal contained in an unbroken set of measurements from a single satellite at a single receiver). The process is referred to as integer ambiguity resolution and its final solution ("fixed") can be done at the mm-cm level (Henning 2011).

\subsection{Integrated GPS and GPR surveys: technical aspects}

The NMEA 0183 communications protocol is most often used for data exchange between GPS signal receivers and other electronic devices. Data from the receiver are sent to the GPR controller (a laptop or a dedicated 

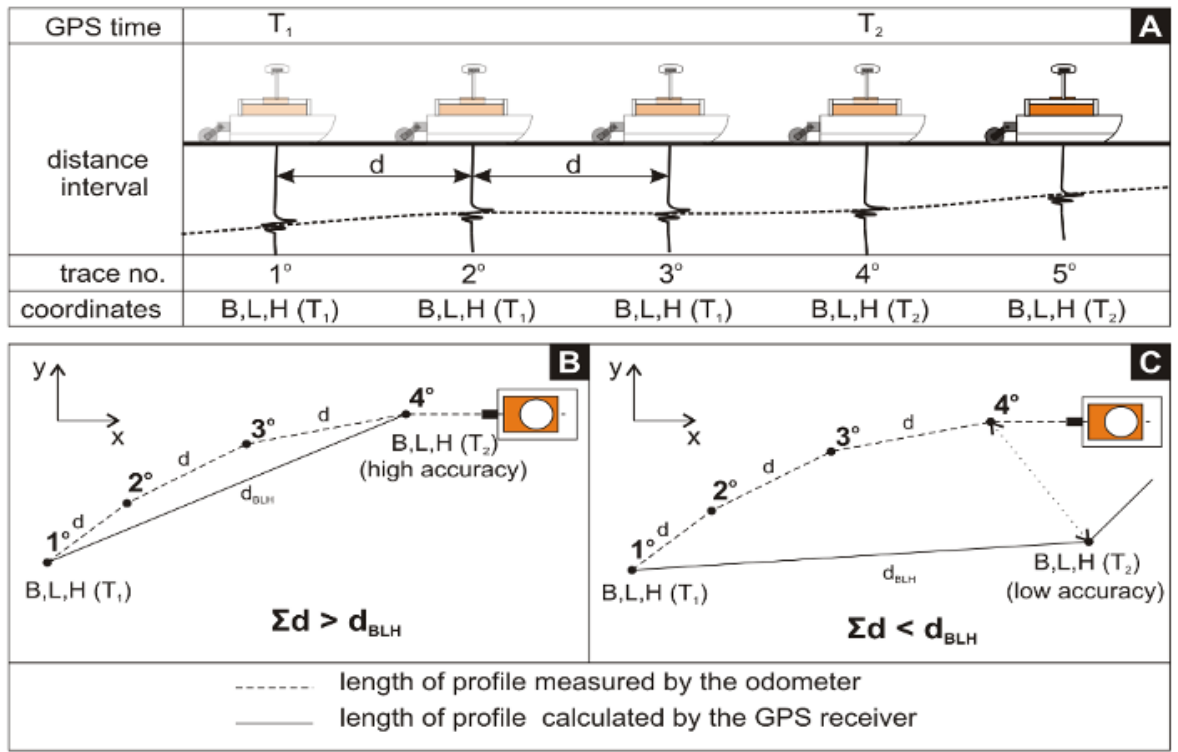

Fig. 1. Schema of the integration of GPS and GPR surveys (A) and errors in determining the length of the profile by a GPS receiver (B) and (C).

monitor) as autonomous sequences of text characters. Each of them contains data on the position (longitude, latitude, and altitude) and time. GPR survey data are also sent to the controller at the same moment. The data acquisition application generates a text file where individual traces, denoted with numbers, are assigned their position and time (Rial et al. 2005, Malå GeoScience 2010). However, this solution has some limitations. The signal in a ground penetrating radar is often released with high frequency and the GPS signal receiver cannot update its position equally quickly. This leads to situations where different traces have the same coordinates. The example is presented in Fig. 1A. Then the distance calculated on the basis of the coordinates may be significantly different from the distance measured by the odometer (examples in Fig. 1B). It depends on the speed of GPR movement the interval distance parameter, frequency of satellite measurements and the object that was measured. But, in many cases, low accuracy of GPS coordinates also affects the miscalculation of the length of the profile (Fig. 1C).

In more recent software versions, it is possible to set position interpolation for traces without determined coordinates (Ortyl and Bałut 2006), but it does not eliminate errors in determination of the distance.

Because of the electromagnetic signal nature, Rial et al. (2005) suggested in their paper that metal fixings and other elements must be avoided. However, Ortyl (2006) demonstrated in his research that a GPS signal re- 
ceiver mounted on a $500 \mathrm{MHz}$ antenna, fixed at around $0.7 \mathrm{~m}$ over the GPR antenna, had no significant effect on the results of ground penetrating radar measurements. It has not yet been studied how signals from GLONASS satellites affect the quality of the obtained GPR data, but because both systems use similar frequencies, the authors assumed that their effect would also be low.

\section{CASE STUDY}

The research was carried out along several kilometers of a dyke on the Łyna River in Olsztyn (north-eastern Poland). This structure was selected because in the past heavy rainfall had caused the river to breach the dyke crown, flood the adjacent areas and block an important local road. The conducted terrain inspection showed that the main cause of the ground surface inundation had been burrows of small fossorial mammals which had been weakening the body of the dyke. Fig. 2 shows an example of animal activity harmful to the construction of the structure. The aim of the ground penetrating radar examination was to provide more detailed information on the condition of the structure and the precise location of the damaged places.

The equipment used in the survey was Malå GPR ProEx System, Malå GeoScience, Sweden, fitted with a $500 \mathrm{MHz}$ antenna and a ProEx Control Unit. This is the most universal antenna used for detecting small objects (a minimum of $5 \mathrm{~cm}$ in diameter) and, depending on the soil conditions, it provides a depth range from 3 to $5 \mathrm{~m}$ (Malå GeoScience 2003). The application Malå GroundVision for ProEx and X3M v. 1.26.1 was used for ground penetrating radar data acquisition. The ground penetrating radar's transmitting antenna released an electromagnetic wave based on the covered distance and measured with an odometer. The distance interval was $0.02 \mathrm{~m}$.

A Trimble R8 SPS882 signal receiver, designed for precise geodetic surveys, was fixed to the ground penetrating radar antenna with a steel bracket

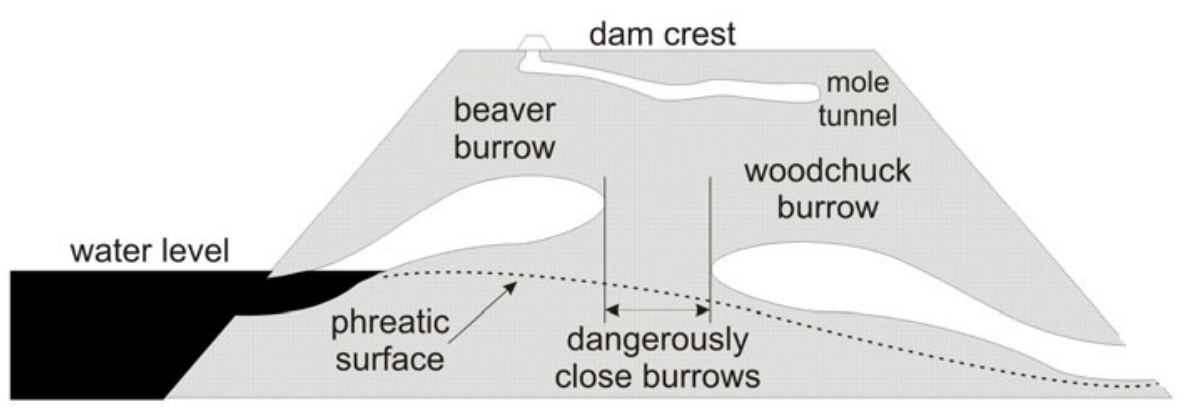

Fig. 2. Impacts of animals on earthen dams (FEMA 2005). 


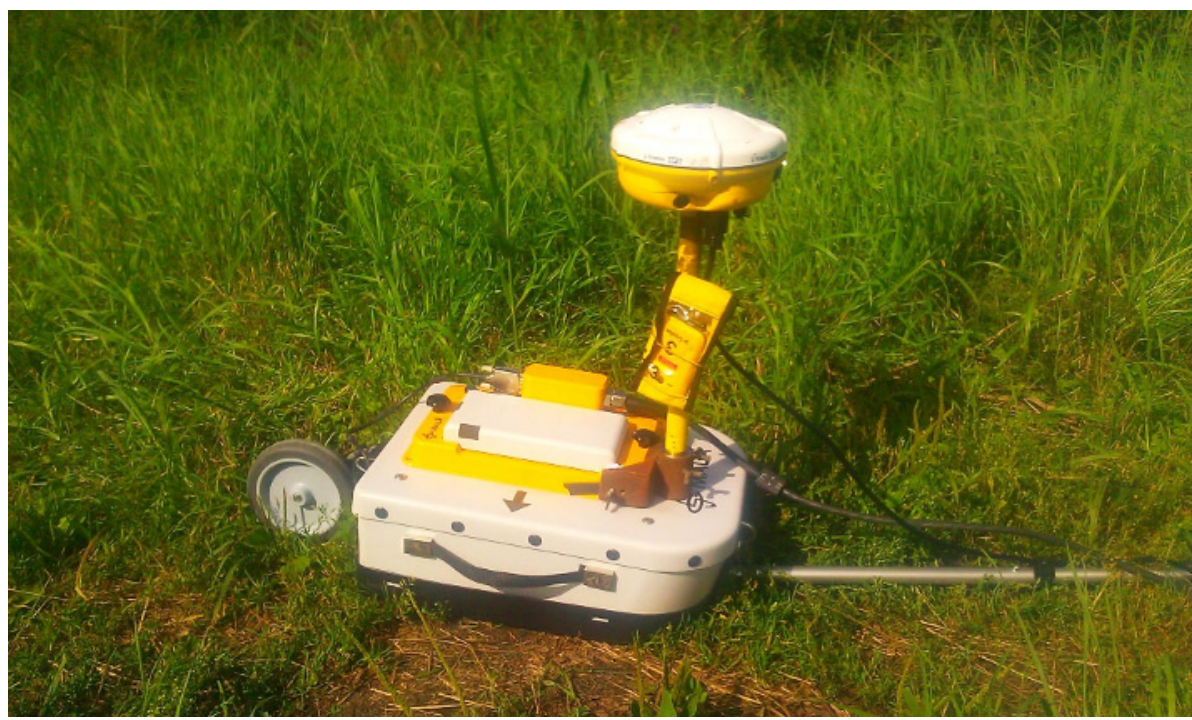

Fig. 3. Integrated survey set (ground penetrating radar antenna and GPS receiver).

and a $50 \mathrm{~cm}$ pole. The receiver operated in RTK survey mode and determined the position based on GPS and GLONASS satellites. One of the stations of the national reference station network ASG-EUPOS, located up to $2 \mathrm{~km}$ from the survey site, was used as the reference receiver. The receiver position determination frequency was $10 \mathrm{~Hz}$. The survey set is shown in Fig. 3.

Reflex-Win 6.0 software was used for data processing and interpretation (Sandmeier 2011). This is an advanced application for filtering ground penetrating radar survey and seismic survey data. In the study the standard functions of the 2D-data-analysis module described in the REFLEXW 6.0 User Manual (Sandmeier 2011) were used. These included the move start-time, subtract mean (dewow), subtract-DC-shift, background removal, gain function, bandpass Butterworth and correct 3D-topography functions. The move start-time algorithm cuts off several samples from the start of the signal to determine the beginning of the depth scale. The dewow filtering removes subsequent decay of "wow" or low-frequency signal trend present in the data and eliminates a long waved part of the signal caused by the electromagnetic induction (Jol 2009). The DC-shift procedure converts ground penetrating radar signals to a form with the zero mean, subtracting their constant component. This enables subsequent correct survey material processing using other functions, i.e., frequency filtering and gain (Karczewski et al. 2011). The "background removal" function removes background noise and reduces 
constant horizontal banding across the radargrams (signal ringing effect) (Goodman 2006, Jol 2009). "Bandpass Butterworth" is one of temporal filters and it removes high frequency noise ("speckle"). "Gain function" improves the data display and interpretation (Jol 2009). It enhances a time slice based on manually set two filter parameters: linear gain and exponent (Sandmeier 2011). The "correct 3D-topography" function is a more advanced tool for trace correction in the time axis direction based on topographic data (Sandmeier 2011). The use of other non-standard procedures resulted from the needs which emerged in the interpretation process.

\subsection{Field experiment description}

The survey was conducted in sections of the levees separating the river from the area of a drained lake. The research object was divided into two parts (see Fig. 4). The first fragment (A) was in the immediate vicinity of a housing estate where animal activity seemed low. The second (B) was distant from human agglomerations. A greater damage caused by the action of small

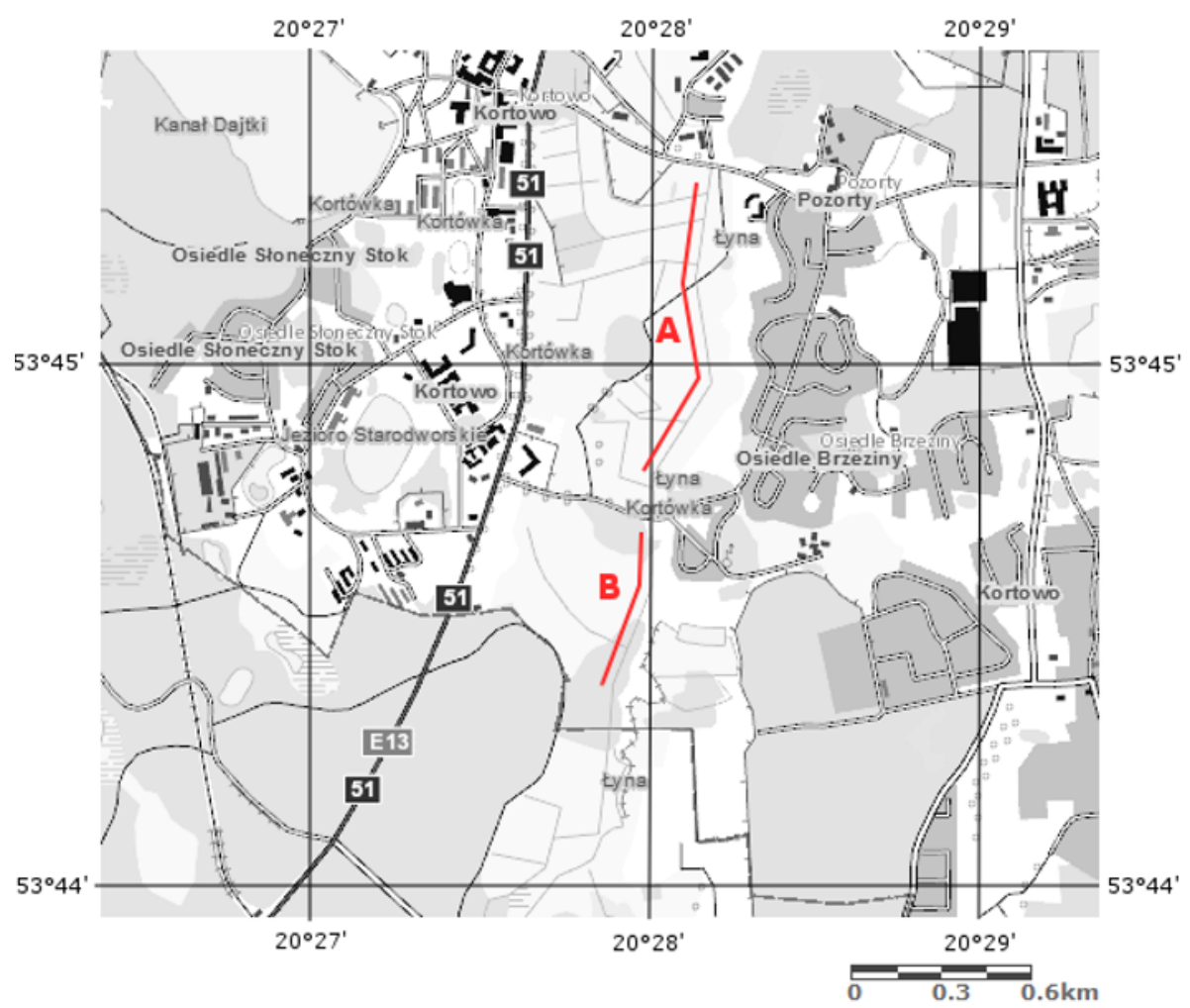

Fig. 4. Distribution map of the measured levee fragments, where (A) means low animals activity expected there, and (B) means high animals activity expected there. 
mammals was expected there. Unlike in Di Prinzio et al. (2010), the research area was not limited to fragments devoid of factors with a negative effect on survey results, such as arborescent vegetation and shrubs, because, in practice, the occurrence of this type of objects in the immediate vicinity of hydraulic engineering structures cannot be excluded, although this is not desirable.

The embankments were built towards the end of the 1950s in the river dredging process. Soil extracted from its bottom was heaped up on the shores of the former body of water, in places where meadows were to be protected against flooding. The dyke width was designed as $2 \mathrm{~m}$, its height as $1.50 \mathrm{~m}$ (altitude $104.21 \mathrm{~m}$ ), and the slope in a 1:1.5 ratio. The levee consists of peat-mud soils, low moor peats, valley peats, and sandy soils with slightly loamy sand. The levees were modernized in the early 1990s. All of their losses resulting from subsidence and breaching were filled in with material from the river bottom and the dyke was then sown with a grass mixture (ZMiUW 1992).

As it has been mentioned in Section 2, it often happens in practice that the examined ground is composed of several materials. Also the variation of water content in the ground could affect the variations of GPR velocity (Sénéchal and Sénéchal 2010). In this case, the wave velocity determined on the basis of the relative dielectric permittivity parameter may be incorrect. Wave velocity in the medium was evaluated on the basis of a diffraction hyperbola analysis and velocity profile verses two-way travel time from hyperbolic matching data. After applying the "time-depth conversion" filter, the antenna's range was around $1.5 \mathrm{~m}$ in depth. The next step was to use "time cut" procedure to clip data after a subjectively identified two-way travel time beyond which noise exceeded the signal.

The terrain conditions in the vicinity of the examined levee were favorable for carrying out a satellite survey. To the west, the horizon was open most of the time and objects which could obstruct satellites (i.e., trees or shrubs) appeared only sporadically. To the east, there were a flowing river, buildings and small clusters of trees and shrubs on a hill which could obscure the satellites at an elevation not higher than 45 degrees. This did not hinder, however, the determination of the receiver's position and the coordinates were determined for all surveyed points. However, a large terrain obstruction in satellite positioning can cause gross errors (Bakuła 2012, 2013).

\section{DISCUSSION OF THE RESULTS}

A set of files containing data on the structure of the levees along $2 \mathrm{~km}$ and a set of files with spatial data assigned to the trace numbers were obtained from the field work. 


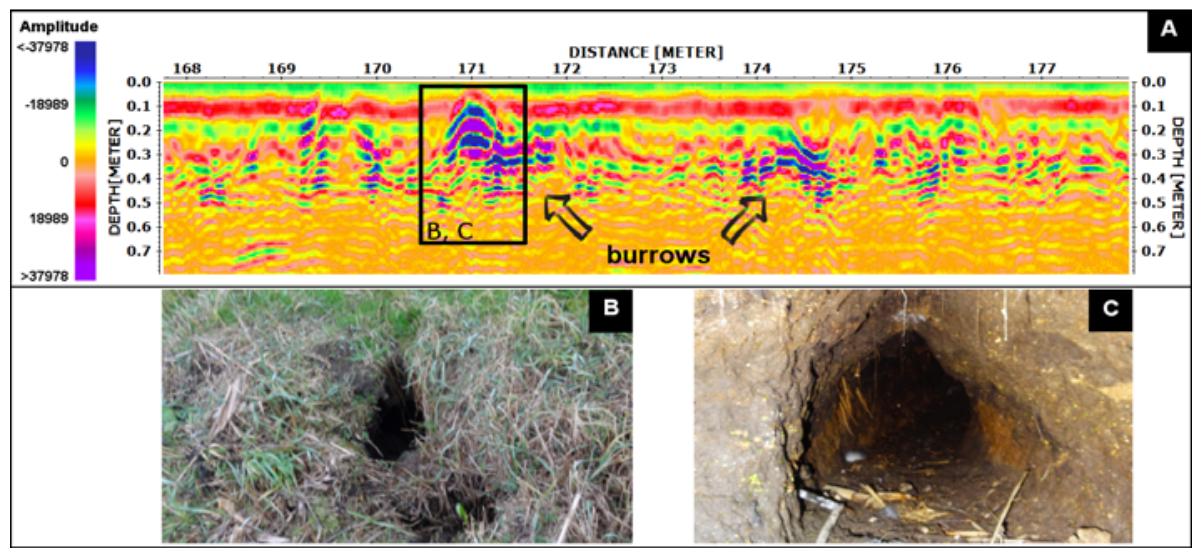

Fig. 5. Tunnel detected during the inspection and its imaging on the radargram (A).

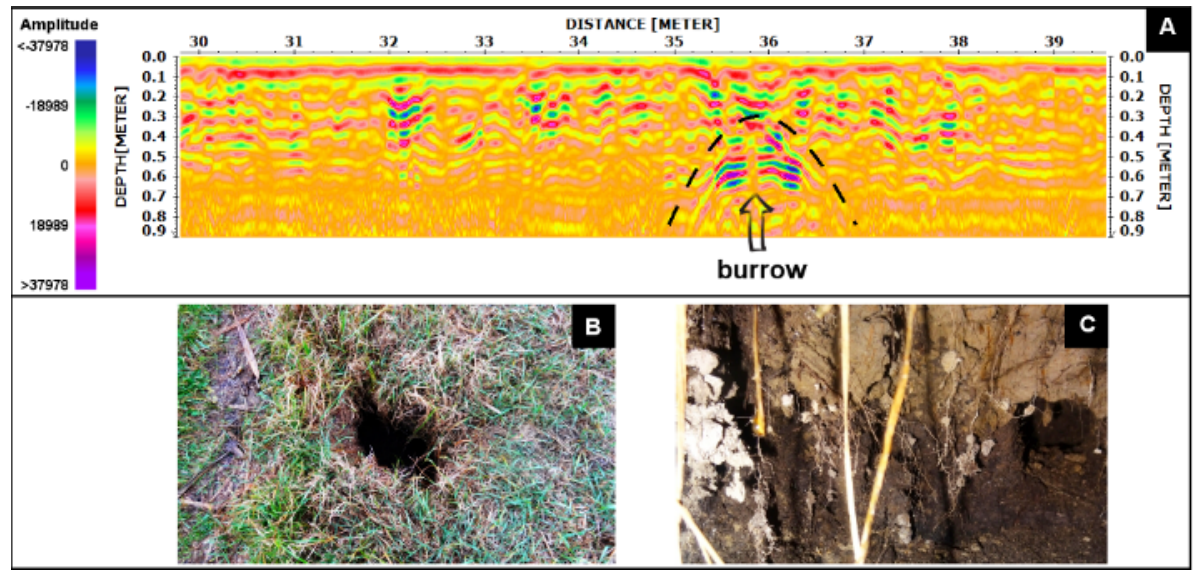

Fig. 6. Tunnel detected after data processing, its imaging on the radargram (A) and photos (B) and (C).

Data processing confirmed the presence of these voids in the levee which had been observed during the inspection of the structure. Figure 5 shows a scan fragment with a visible prelocated burrow at the depth of $10 \mathrm{~cm}$. About $3 \mathrm{~m}$ away there was another tunnel, which had not been detected before the measurements. Figure 6 shows the image of the next burrow. It had not been detected during the earlier visual inspection and the survey. These anomalies could be identified only after data processing. These anomalies could be identified only after data processing. Based on their coordinates, places were found in the field and small excavations were made there, which confirmed our suppositions as to the cause of signal interference. 


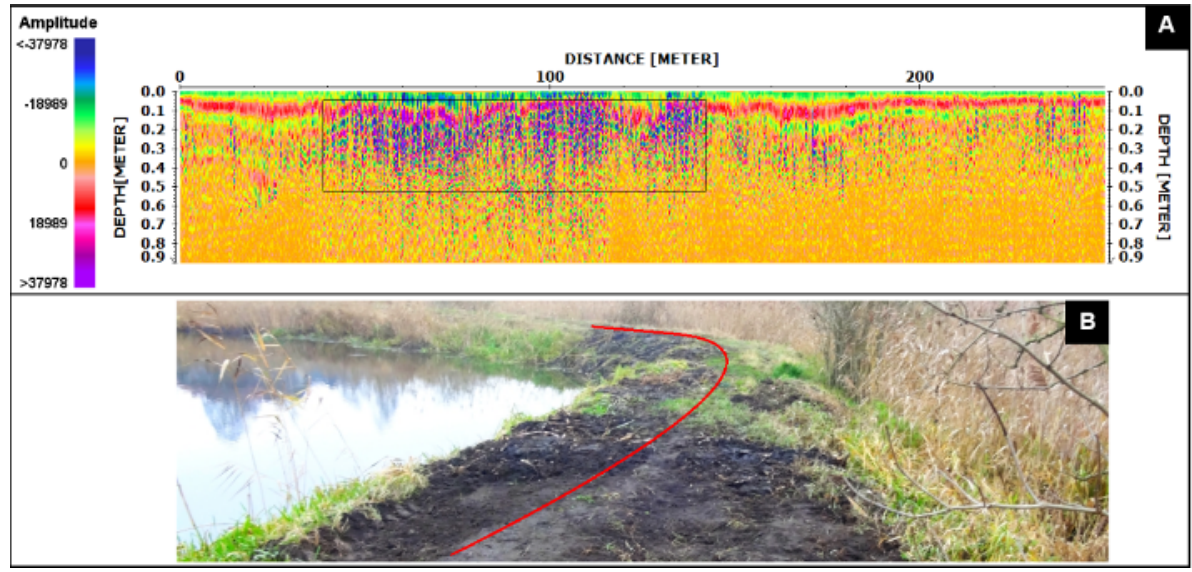

Fig. 7. Repaired levee section, its imaging on the radargram (A) and its photo (B).

Additionally, Fig. 7 presents a radargram fragment with a visible section of distinct signal interference extending from 40 to $140 \mathrm{~m}$. This is the place where the levee had been destroyed by the river. It was repaired using sandbags and material from the river bottom immediately after this event.

The roots of large shrubs growing on the levee banks and collapsed tunnels often disturbed survey data and, in some cases, it was difficult to determine whether they were the cause of the present anomalies or whether it should be sought in other factors. In the places where the levee was flat and free from vegetation, the results were satisfactory.

All anomalies, which were considered as objects with a negative effect on the levee structure, were denoted with markers. A point set was obtained from the accurate GPS survey in the way it can be imported in applications for generating digital maps and Geographic Information Systems. The example was shown in Fig. 8A, where the blue dots are the equivalent of burrows detected during GPR survey. Moreover, the results of the measurement are used as input data in the filter correct 3D-topography.

An accuracy analysis of profiles length determination by the satellite survey was also performed. For this purpose, the distance travelled by the receiver during the survey at individual route sections was computed and compared to the distance recorded by the odometer of the ground penetrating radar equipment. A single profile consisted of around from 1500 to 2000 traces with measured coordinates. The results of these comparisons $\left(\Delta_{1}\right)$ are shown in Table 2. The profiles with the numbers 1-5 compose the distance covered in the first direction and numbers 6-10 in the return direction. 


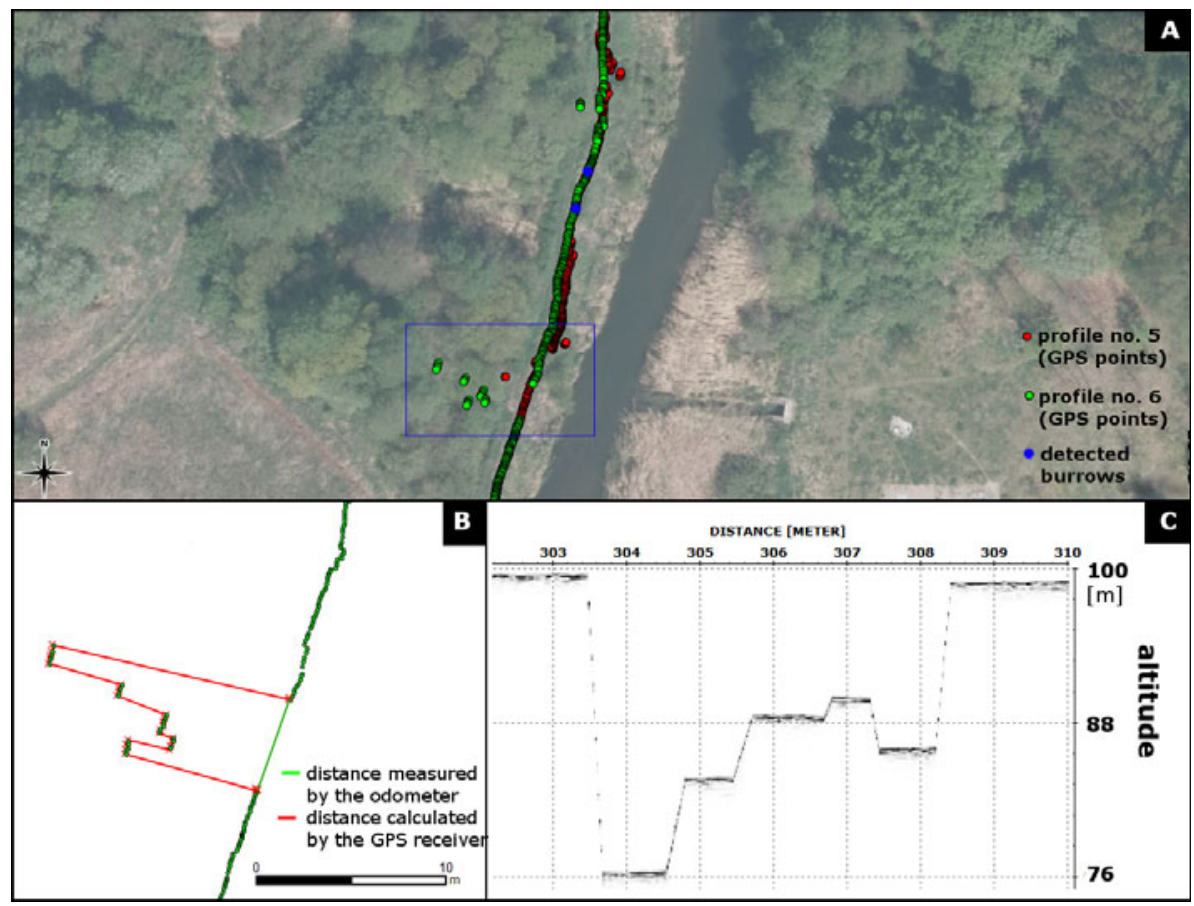

Fig. 8. Gross errors in determined coordinates and altitude (A) and their effect on calculated distance (B) and correct 3D-topography filter (C).

Table 2

Differences between the distance

measured with the ground penetrating radar equipment and with the GPS receiver before $\left(\Delta_{1}\right)$ and after $\left(\Delta_{2}\right)$ coordinates correction

\begin{tabular}{|c|c|c|c|c|c|}
\hline $\begin{array}{c}\text { Profile } \\
\text { no. }\end{array}$ & $\begin{array}{c}\text { GPR distance } \\
{[\mathrm{m}]} \\
(1)\end{array}$ & $\begin{array}{c}\text { GPS distance } \\
{[\mathrm{m}]} \\
(2)\end{array}$ & $\begin{array}{c}\Delta_{1} \\
{[\mathrm{~m}]} \\
(2)-(1)\end{array}$ & $\begin{array}{c}\text { Corrected } \\
\text { GPS distance } \\
\text { (3) }\end{array}$ & $\begin{array}{c}\Delta_{2} \\
{[\mathrm{~m}]} \\
(3)-(1)\end{array}$ \\
\hline 1 & 202.25 & 280.73 & 78.48 & 217.48 & 15.23 \\
\hline 2 & 200.25 & 205.87 & 5.62 & 205.62 & 5.37 \\
\hline 3 & 250.08 & 268.48 & 18.40 & 259.43 & 9.35 \\
\hline 4 & 251.16 & 259.59 & 8.43 & 257.81 & 6.65 \\
\hline 5 & 336.77 & 377.02 & 40.25 & 347.22 & 10.45 \\
\hline 6 & 360.34 & 403.30 & 42.96 & 370.04 & 9.70 \\
\hline 7 & 267.85 & 278.50 & 10.65 & 278.31 & 10.46 \\
\hline 8 & 250.09 & 266.30 & 16.21 & 260.05 & 9.96 \\
\hline 9 & 200.45 & 214.70 & 14.25 & 210.00 & 9.55 \\
\hline 10 & 159.51 & 174.50 & 14.99 & 171.00 & 11.49 \\
\hline
\end{tabular}


Large values of differences, especially for profiles 1,5 , and 6 , indicate gross errors in the determination of distance. This could be caused by failure of the odometer or low accuracy of the designated coordinates. First, odometer has been tested during the measurement profile of a known length. The results of the tests showed no malfunction.

The next step was to control the accuracy of the determination of the coordinate for each of the GPR profiles. The analysis was made on the basis of satellite images and it was set as a background for the determined GPS points. It was noted that the points with low accuracy of the coordinates (standard errors of several meters) were most often near trees and shrubs (Fig. 8A). Trees and other obstacles prevented receiving the signals from satellites and determination high-accuracy three-dimensional coordinates. To obtain the fixed solutions of integer ambiguity during RTK/GPS surveys, at least 5 satellites must be tracked and the quality of signals from satellites also has to be favorable (especially in this method of GPS measurement) (Lee and Ge 2006), so a good condition of measurements is very important.

Sometimes the accuracy of the points was also lower (a dozen or a few tens of centimeters) in places located away from high objects. It could depend on the discontinuities in the time series of carrier-phase measurements due to the receiver temporarily losing lock on the carrier of a GPS signal, known as "cycle slips" (Karaim et al. 2013, Kim and Langley 2001, Hofmann-Wellenhof et al. 1997). Hofmann-Wellenhof et al. (1997) distinguished three causes of cycle slips: obstructions of the satellite signal due to some objects (for example: trees, buildings, mountains); a low signal-tonoise ratio or carrier-to-noise-power-density ratio due to bad ionospheric conditions, signal multipath, high receiver dynamics or low satellite elevation angle and a failure in the receiver software which leads to incorrect signal processing. Then value of a phase ambiguity is determined incorrectly on one or more carrier signals and the coordinates have a lower accuracy.

When the horizontal coordinates have low accuracy, then the attitude is also determined with the gross error (usually much larger than the horizontal coordinates). Thus, the correct 3D-topography filter should not be applied to such satellite surveys as the topography will be inconsistent with the reality, as shown in Fig. 8C.

Based on the analysis of the satellite images and text files with coordinates $(*$. COR files) points with low accuracy were removed. Then the elimination criteria were used:

$\square$ points that are outside the flood embankment and/or are located far away from the other points,

$\square$ points with the value of the altitude that is significantly different from the approximate altitude of the flood embankment (more than one meter). 
After deleting erroneous data, the distance GPS was recalculated and compared with the results of the odometer. The results $\left(\Delta_{2}\right)$ are shown in Table 2. In most cases, the results are better, although they are still not perfect. Besides, the GPR traces for which the coordinates were removed have interpolated BLH values. They also can be calculated with an error (smaller, but difficult to estimate).

So which option ought to be chosen: inaccurate coordinates and the knowledge that they cannot be used to locate anomalies or interpolated values of the coordinates of the unknown accuracy? The authors believe the answer to this question depends on the purpose of surveys. In this case, we recommend using the original coordinates. The authors suggest measuring the profiles twice as one of them is likely to have a better set of BLH values. But in the case of GPR measurements using low-frequency antennas, coordinate interpolation errors will have no meaning.

\section{CONCLUSION}

The results of the survey prove that the GPR method can be used for monitoring the condition of hydraulic engineering structures. Data processing results shown in Figs. 4-6 illustrate examples of interference in the structure of the examined ground. In the first two cases, these are tunnels dug by small mammals. In the third, high disturbance was caused the repair work recently being performed on the levee. That supports Di Prinzio et al. (2010) that in places where the levee underwent any changes, the received signals differ from those recorded where the structure was intact.

Since the GPR method is sensitive to interference caused by various geologic and cultural factors (like reflections from nearby vehicles, buildings, fences, power lines, and trees), unprocessed radargrams could provide only approximate shapes and depths. That means some anomalies in the examined structure may be noticed only after data processing. In this situation, GPR data integration with satellite measurements indicates the importance of data processing. Only because accurate geodetic coordinates are assigned to individual traces, they can be located later in the field, plotted on a map and fed into a Geographic Information System.

Nevertheless, the most accurate real-time kinematic surveys, which are commonly used in the geodesy, are not ideal and error-free. Their limitation may be a too small number of observed satellites, a cycle slip and loss of access to the observations sent from the base station. They cause errors in determining the coordinates and height, which do not allow for the correct application of GPS data to locate anomalies and topography modeling during GPR data processing. The solution may be a two-time measurement of one profile or data analysis and removal of inaccurate GPS coordinates. The choice depends on the purpose of measurements. The solution may be a 
double measure of the profile or GPS data analysis and removal of inaccurate coordinates. The choice depends on the purpose of GPR surveying.

Precise topographic data are important for levee condition examination. According to the technical documentation, the height of the examined structure was gradually decreasing owing to ground subsidence. In the early 1990s, its height was increased with additional embankments. Having available altitude data at individual points of the levee crown, it can be determined in which place the levee is the lowest and requires repair work. This also allows to predict where the river will overflow the levee and which areas are liable to flooding.

Summing up, the comprehensiveness of integrated GPR and GPS surveys in levee monitoring requires to be stressed. They allow data on levee condition to be acquired quickly and economically without interference to its structure, and all damage is located in a coordinate system. In situations where human life and safety are involved, all information is vital and precise determination of weakened places in the body of the levee is priceless knowledge. Therefore, it is worth employing all technologies which significantly improve the safety. Nevertheless, for public services to be able to use them fully, they must provide optimal conditions for conducting satellite and ground penetrating radar surveys. Trees and shrubs which grow on the levee crown not only prevent correct GPR/GPS measurement, but also weaken the structure.

It must also be remembered that the result of a ground penetrating radar survey is full, however general, information on the condition of the examined structure. According to the "from the whole to the part" principle, boreholes and probings should be made in weakened places to obtain complete and necessary data for correct evaluation of the condition of the levee.

Acknowledgements. The authors are grateful to Mr. Jerzy Dziurdź from the Board of Land Amelioration and Water Facilities (Olsztyn, Poland) for making the technical documentation available. The review comments provided by two anonymous reviewers helped to improve the quality of this paper.

Bakuła, M. (2012), An approach to reliable rapid static GNSS surveying, Surv. Rev. 44, 327, 265-271, DOI: 10.1179/1752270611Y.0000000038.

Bakuła, M. (2013), Study of reliable rapid and ultrarapid static GNSS surveying for determination of the coordinates of control points in obstructed conditions, 
J. Surv. Eng. ASCE 139, 4, 188-193, DOI: 10.1061/(ASCE)SU.1943-5428. 0000109.

Borys, M. (2013), Principles of the yearly flood embankments technical state controls realisation, Gospod. Wodna 2013, 7, 260-266 (in Polish).

Bruce, D.A. (1993), A review of drilling and grouting methods for existing embankment dams, Geotech. Spec. Publ. 35, 803-819, DOI: 10.1061/ 9780784412350.0107.

Daniels, D.J. (ed.) (2004), Ground Penetrating Radar, IET Radar, Sonar, Navigation and Avionics Series, Vol. 15, The Institution for Electrical Engineers, London.

Daniels, J.J. (2000), Ground penetrating radar fundamentals. Prepared as an appendix to a report to the U.S.EPA, Region V.

de Vries, S., R.A. Verheij, P.P. Groenewegen, and P. Spreeuwenberg (2003), Natural environments - healthy environments? An exploratory analysis of the relationship between greenspace and health, Environ. Plann. A 35, 10, 1717-1731, DOI: 10.1068/a35111.

Di Prinzio, M., M. Bittelli, A. Castellarin, and P. Rossi Pisa (2010), Application of GPR to the monitoring of river embankments, J. Appl. Geophys. 71, 2-3, 53-61, DOI: 10.1016/j.jappgeo.2010.04.002.

Doocy, S., A. Daniels, S. Murray, and T.D. Kirsch (2013), The Human impact of floods : a historical review of events 1980-2009 and systematic literature review, PLoS Curr. Disasters 5, PMC3644291, DOI: 10.1371/currents.dis. f4deb457904936b07c09daa98ee8171a.

EC (2004), Flood risk management, flood prevention, protection and mitigation, Communication from the Commission to the Council, the European Parliament, the European Economic and Social Committee and the Committee of the Regions, COM/2004/0472, 11 pp.

EC (2007), Directive 2007/60/EC of the European Parliament and of the Council of 23 October 2007 on the assessment and management of flood risks, Official Journal of European Union on 6 November 2007, L 288/27, 27-24.

Edwards, S.J., P.J. Clarke, N.T. Penna, and S. Goebell (2010), An examination of network RTK GPS services in Great Britain, Surv. Rev. 42, 316, 107-121, DOI: $10.1179 / 003962610 X 12572516251529$.

FEMA (2005), Technical manual for dam owners. Impacts of Animals on Earthen Dams, FEMA 473, Federal Emergency Management Agency.

Fisher, S.C., R.R. Stewart, and H.M. Jol (1992), Processing ground penetrating radar (GPR) data, CREWES Res. Rep. 4, 1-21.

Gołębiowski, T., S. Tomecka-Suchoń, and J. Farbisz (2012), Use of geophysical methods for non-destructive detection and monitoring of technical level in the river embankments. In: European Symp. "Anti-flood Defences - Today's Problems”, 28-30 March 2012, Paris-Orleans, France (in Polish). 
Goodman, D. (2006), GPR Survey at Ft Frederica, Georgia. National Park Service Workshop, May 16-20, 2006, Geophysical Archaeometry Laboratory, Woodland Hills, USA.

Henning, W. (2011), User guidelines for single base real time GNSS positioning, Ver. 2.1, National Oceanic and Atmospheric Administration, National Geodetic Survey, USA.

Hofmann-Wellenhof, B., H. Lichtenegger, and J. Collins (1997), GPS. Theory and Practice, 4th ed., Springer, Wien.

Jol, H.M. (2009), Ground Penetrating Radar: Theory and Applications, Elsevier Science, Amsterdam.

Karaim, M.O., T.B. Karamat, A. Noureldin, M. Tamazin, and M.M. Atia (2013), Real-time cycle-slip detection and correction for land vehicle navigation using inertial aiding. In: Proc. 26th Int. Technical Meeting of the ION Satellite Division, ION GNSS+ 2013, 16-20 September 2013, Institute of Navigation, Nashville, USA.

Karczewski, J. Ł. Ortyl, and M. Pasternak (2011), Zarys Metody Georadarowej, Wydawnictwa AGH, Kraków (in Polish).

Kim, D., and R.B. Langley (2001), Instantaneous real-time cycle-slip correction of dual-frequency GPS data. In: Proc. Int. Symp. on Kinematic Systems in Geodesy, Geomatics and Navigation KIS 2001, 5-8 June 2001, Banff, Canada, 255-264.

Lee, D.-H., J.-H. Wu, H.-M. Lin, P.-M. Liao, G.-Z. Zeng, G.-C. Yang, and C.-J. Liao (2012), Classification of the eroded cavities behind concretefaced embankments by ground penetration radar. In: Proc. 6th Int. Conf. on Scour and Erosion, 27-31 August 2012, Paris, France, 1393-1399.

Lee, I.-S., and L. Ge (2006), The performance of RTK-GPS surveying under challenging environmental conditions, Earth Planets Space 58, 5, 515-522, DOI: 10.1186/BF03351948.

Malå GeoScience (2003), Antenna $500 \mathrm{MHz}$ for ground penetrating radar investigation. User Manual, Malå GeoScience, Sweden.

Malå GeoScience (2010), Using Malå GPR systems with GPS equipment, Application note, Printed Matter No. 2894, Malå GeoScience, Sweden, 1-5.

Morey, R.M. (1998), Ground Penetrating Radar for Evaluating Subsurface Conditions for Transportation Facilities, NCHRP Synthesis 255, Transportation Research Board, National Research Council, National Academy Press, Washington.

Mori, G. (2009), The use of Ground Penetrating Radar and alternative geophysical techniques for assessing embankments and dykes safety, Ph.D. Thesis, Università di Bologna, Bologna, Italy.

Mydlikowski, R., and G. Beziuk (2009), Examination of levee condition by means of GPR, Studia Geotech. Mech. 31, 4, 49-56. 
Mydlikowski, R., and A. Szynkiewicz (2009), Non-invasion detection inhomogeneities in structure of levees by radar GPR, Trans. VSB - Tech. Univ. Ostrava 9, 2, 178-192.

Niederleithinger, E., A. Weller, R. Lewis, U. Stötzner, T. Fechner, B. Lorenz, and J. Niessen (2008), Evaluation of geophysical methods for river embankment investigation. In: Proc. 4th Int. Symp. on Flood Defence: Managing Flood Risk, Reliability and Vulnerability, Toronto, Ontario, Canada.

Ortyl, Ł. (2006), Examination of influence of a GPR-GPS measuring set external elements on results of georadar sensing, Geodesy 42, 45-60.

Ortyl, Ł., and A. Bałut (2006), Application of a RTK-GPS technique in the process of 3-D location of georadar profile traces, Geodesy 42, 27-44.

Rial, F.I., M. Pereira, H. Lorenzo, and P.Arias (2005), Acquisition and synchronism of GPR and GPS data: application on road evaluation, Image Signal Process. Remote Sens. 9, 372-379, DOI: 10.1117/12.627965.

Sandmeier, K.J. (2011), ReflexW version 6.0. User manual, Software Manual, Karlsruhe, Germany, 532 pp.

Sénéchal, P., and G. Sénéchal (2010), Relationships between water flow rate and geophysical measurements in an alluvial aquifer, Acta Geophys. 58, 1, 8395, DOI: 10.2478/s11600-009-0024-7.

Smith, D.G., and H.M. Jol (1995), Ground penetrating radar: antenna frequencies and maximum probable depths of penetration in Quaternary sediments, J. Appl. Geophys. 33, 1-3, 93-100, DOI: 10.1016/0926-9851(95)90032-2.

Song, H., and Z. Guo (2010), Ground penetrating radar model simulation for detection of coastal embankment, Electron J. Geotech. Eng. 15, 589-600.

Szynkiewicz, A. (2000), GPR monitoring of earthen flood banks/levees. In: D.A. Noon, G.F. Stickley, and D. Longstaff (eds.), Proc. SPIE 4084, Eighth International Conference on Ground Penetrating Radar, 23 May 2000, Gold Coast, Australia, 85-90, DOI: 10.1117/12.383541.

Tillard, S., and J.C. Dubois (1995), Analysis of GPR data: wave propagation velocity determination, J. Appl. Geophys. 33, 1-3, 77-91, DOI: 10.1016/09269851(95)90031-4.

Urbini, S., L. Vittuari, and S. Gandolfi (2001), GPR and GPS data integration: examples of application in Antarctica, Ann. Geophys. 44, 4, 687-702, DOI: 10.4401/ag-3568.

ZMiUW (1992), Construction of levees on the Lyna river, Technical Project, Board of Land Reclamation and Water Facilities (ZMiUW), Warsaw, Poland (unpublished).

Received 20 January 2014 Received in revised form 24 October 2014 Accepted 6 August 2015 